\title{
PRECISE POSE RECOVERY OF DISTAL LOCKING HOLES FROM SINGLE CALIBRATED FLUOROSCOPIC IMAGE VIA A NOVEL VARIABLE DECOMPOSITION APPROACH
}

\author{
Xuan Zhang and Guoyan Zheng \\ MEM Research Center - ISTB, Stauffacherstrasse 78, CH-3014, Bern, Switzerland
}

\begin{abstract}
This paper presents a novel variable decomposition approach for pose recovery of the distal locking holes using single calibrated fluoroscopic image. The problem is formulated as a model-based optimal fitting process, where the control variables are decomposed into two sets: (a) the angle between the nail axis and its projection on the imaging plane, and (b) the translation and rotation of the geometrical model of the distal locking hole around the nail axis. By using an iterative algorithm to find the optimal values of the latter set of variables for any given value of the former variable, we reduce the multiple-dimensional model-based optimal fitting problem to a one-dimensional search along a finite interval. We report the results of our in vitro experiments, which demonstrate that the accuracy of our approach is adequate for successful distal locking of intramedullary nails.
\end{abstract}

Index Terms-Biomedical applications of X-ray, bones, parameter estimation, optimization methods

\section{INTRODUCTION}

One of the most difficult steps of intramedullary nailing of femoral shaft fractures is distal locking - the insertion of distal interlocking screws, for which it is necessary to know the positions and orientations of the distal locking holes (DLHs) of the intramedullary nail (IMN). Complicating the process of locating and inserting the distal interlocking screw is the nail deformation with insertion. It has been reported that deformation occurs in several planes due to medial-lateral (ML) and anterior-posterior (AP) flexion of the distal nail after it has been inserted [1]. Therefore, it is very difficult, to determine what the resultant locations and orientations of the DLHs will be relative to their initial position before it is deformed. The surgeon depends heavily on intra-operative X-ray means in a conventional surgical procedure for providing precise locations and orientations of the DLHs. It requires positioning the axis of the fluoroscope perpendicular to the locking holes so that these holes appear perfectly circular in the images. This is achieved through a trial-and-error method and requires long time X-ray exposure for both the surgeon and patient. It has been reported that the surgeon's exposure to radiation for each conventional surgical procedure was $3-30 \mathrm{~min}$, of which $31 \%-51 \%$ was used for distal locking [2].

The desire to target accurately with as little as possible $\mathrm{X}$-ray exposure has led to various attempts to develop image-based methods for recovering the positions and orientations of DLHs [3][4][5][6]. These methods require either multiple calibrated images or single image but with perfectly circular holes in the image, which normally requires the X-ray technician to use a try-and-move method several times to achieve.

This paper presents a novel variable decomposition approach for solving this problem using single calibrated fluoroscopic image. We do not ask for an image with perfectly circular holes but we do put a constraint on its acquisition [6], i.e., the reduced patient shaft should be roughly parallel to the image intensifier (II) of the fluoroscopy machine. We then formulate the pose recovery of the DLH as a model-based fitting problem and decompose the control variables into two sets: (a) the angle between the nail axis and its projection on the imaging plane, and (b) the translation and rotation of the geometrical models of the DLHs around the nail axis. By using an iterative closest projection point algorithm introduced in $[5,6]$ to find the optimal values of the latter set of variables for each give value of the former variable, we reduce the multiple-dimensional optimal fitting problem to a one-dimensional search along a finite interval.

\section{IMAGE CALIBRATION, GEOMETRICAL MODELS, AND PREPROCESSING}

Image Calibration: In reality, the proximal fragment, the distal fragment, and the nail may be treated as three rigid bodies and registered independently. The rigid transformations between these three rigid bodies can be trivially obtained from a navigator such as an optoelectronic tracker, a magnetic tracker, or even a medical robot. As this is not our focus in this paper, here we assume that the fractured femur has already been reduced and the proximal fragment and distal fragment are kept fixed relative to each other at the time of image 
acquisition. We also assume that the nail has been inserted till the distal end of the femur and has been locked proximally by screw so that the complete femur and the nail can be treated as one rigid body. A local coordinate system (COS) is established on this rigid body through a so-called dynamic reference base (DRB) technique. In the following description, let's denote this patient COS as A-COS. All computations are done in this reference COS.

To relate a pixel in the two-dimensional (2D) projection image to $A-C O S$, the acquired image has to be calibrated for physical projection properties and be corrected for various types of distortion. We have chosen a weak-perspective pin-hole camera model for modeling the $\mathrm{C}$-arm projection [7]. Here we assume that for each pixel in the input image we can always find a projection ray emitting from the focal point of the image through the pixel and a three-dimensional (3D) intersection point of between its associated projection ray and the imaging plane

Geometrical Models: The distal part of IMN containing the two DLHs, which is what we are interested in, is modeled as a cylinder (Figure 1, left). The distance $L$ between the centers of the two DLHs can be accurately extracted from its product information. The geometrical model of the DLH is represented by two circles as shown by Figure 1, right, and is used later to simulate X-ray projection of the DLH model.

To obtain the coordinates of those points (visualized as red dots in Figure 1, right) used to describe the model of the DLH, a local COS $C^{\prime} u v w$ is established by taking the intersection point $C$ (it is also called the center of the DLH) between the axis of the DLH and the axis of the IMN as the origin, the axis of the IMN as the $u$ axis, and the axis of the DLH as the $v$ axis (see Figure 1 for details). The coordinates of those points expressed in this local COS can be directly measured from the nail using a caliber, thanks to the symmetrical property of the DLH; or extracted from the engineering drawings of the nail, if they are available.

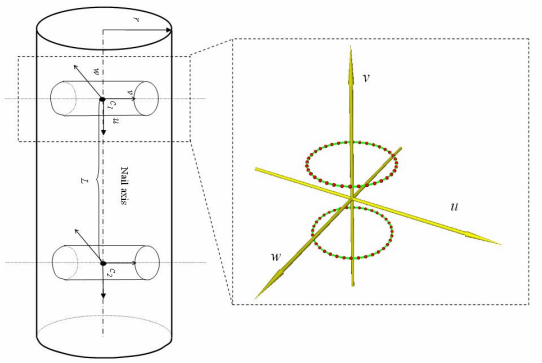

Figure 1. The geometrical model of the distal part of the IMN and the geometrical model of the distal locking hole .

Preprocessing: The task of the preprocessing is to determine the projection points of the centers of the DLHs. To extract these feature points from the image, Hough transform [8] is used to find the two mostly parallel edge lines of the projection of the distal part of the IMN after applying a Canny edge detector to the image. The projection of the axis of the distal part of the IMN is considered as the middle line between these two mostly parallel edge lines. To determine those edge pixels belonging to DLHs, the method reported in [3] is modified for our purpose. A parallelpiped window, whose sizes are equal to the distance between the detected edge lines, is swept along the middle line to find two locations which contain the maximum number of edge pixels and whose distance is greater than a pre-selected distance threshold $T$ (e.g. the width of the window). The centroids of the detected edge pixels in both locations are then calculated. The projection point of the center of each DLH is then determined by finding the closest point on the middle line to the associated centroid. An example of feature point detection is shown in Figure 2.

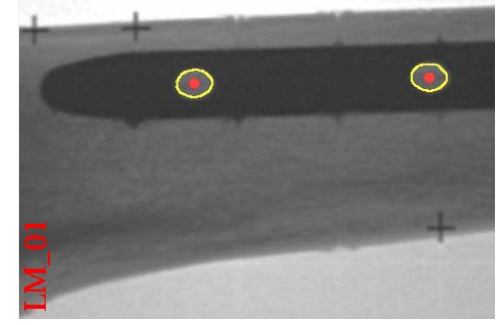

Figure 2. Feature point detection. The detected projection points (red dots) of the centers of both DLHs are displayed together with the edge pixels of the DLHs (yellow)

\section{THE PROPOSED APPROACH}

\subsection{Model-based Fitting for pose recovery}

Using above detected feature points, we can find their corresponding spatial points on the imaging plane. Let's denote them as $d_{l}$ corresponding to the projection point of the center $C_{l}$ of the distal DLH (the DLH that is closer to the nail tip), and $d_{2}$ corresponding to the projection point of the center $C_{2}$ of the proximal DLH, respectively, as shown in Figure 3. These two points define a line in A-COS. This line together with the focal point $f$ defines a plane where the axis of the distal part of the nail should fall in. As we know the coordinates for point $f, d_{l}$, and $d_{2}$, we can calculate three internal angles $\omega_{1}, \omega_{2}$, and $\omega_{3}$ of triangle $f d_{1} d_{2}$. Assume the angle between the nail axis and its projection in the imaging plane is $\alpha$, then the coordinates of the centers of both DLHs are calculated as following:

$$
\begin{aligned}
& C_{1}=f+L_{1} \cdot \frac{\left(d_{1}-f\right)}{\left\|d_{1}-f\right\|} ; \quad C_{2}=f+L_{2} \cdot \frac{\left(d_{2}-f\right)}{\left\|d_{2}-f\right\|} \\
& L_{1}=L \cdot \frac{\sin \left(\omega_{2}+\alpha\right)}{\sin \left(\omega_{3}\right)} \\
& L_{2}=L \cdot \frac{\sin \left(\omega_{2}+\alpha\right) \cdot \cos \left(\omega_{3}\right)}{\sin \left(\omega_{3}\right)}+L \cdot \cos \left(\omega_{2}+\alpha\right) \\
& \text { where } \quad \alpha \in(-\pi / 2, \pi / 2)
\end{aligned}
$$


where $L$ is the distance between the centers of two DLHs. It can be measured or extracted from the product information.

Accoding to equation (1), the coordinates of both centers only depends on the parameter $\alpha$, so as the direction of the nail axis $\left(n_{x}, n_{y}, n_{z}\right)$.

Assuming that the coordinates of the center $C$ of one of the DLHs is denoted as $\left[C_{x}, C_{y}, C_{z}\right]^{T}$, the problem to estimate the pose of the DLH in $A-C O S$ is now changed to find the rotation angle $\alpha$, rotation angle $\theta$, and translation distance $\delta$ of the geometrical model of the DLH along the nail axis $\left[n_{x}, n_{y}, n_{z}\right]^{T}$ so that the simulated X-ray projection of the DLH can be fitted to its real X-ray projection (see Figure 3). This constrained transformation around the parameterized nail axis could be derived as in $[5,6]$ :

$\operatorname{rot}(\alpha, \theta, \delta)=\left[\begin{array}{ccc}n_{x}^{2}+\left(n_{y}^{2}+n_{z}^{2}\right) \cos (\theta) & n_{x} n_{y}(1-\cos (\theta))-n_{z} \sin (\theta) & n_{x} n_{z}(1-\cos (\theta))+n_{y} \sin (\theta) \\ n_{x} n_{y}(1-\cos (\theta))+n_{z} \sin (\theta) & n_{y}^{2}+\left(n_{x}^{2}+n_{z}^{2}\right) \cos (\theta) & n_{y} n_{z}(1-\cos (\theta))-n_{x} \sin (\theta) \\ n_{x} n_{z}(1-\cos (\theta))-n_{y} \sin (\theta) & n_{y} n_{z}(1-\cos (\theta))+n_{x} \sin (\theta) & n_{z}^{2}+\left(n_{x}^{2}+n_{y}^{2}\right) \cos (\theta)\end{array}\right]$

and $\operatorname{trans}(\alpha, \theta, \delta)=\left[t_{x}, t_{y}, t_{z}\right]^{T} ;$ where $t_{x}, t_{y}, t_{z}$ are defined as;

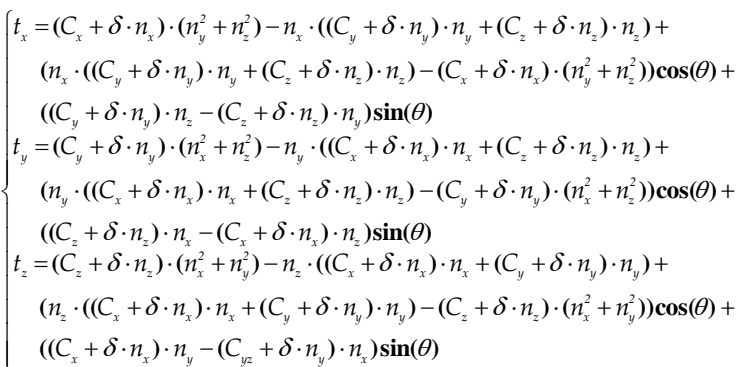

The pose recovery problem can then be formulated as a optimal model-based fitting as in [5, 6]:

$$
\min _{\left\{\alpha^{*}, \theta^{*}, \delta^{*}\right\}} \sum_{i}\left\|e_{j=N(i)}-P\left(\operatorname{rot}(\alpha, \theta, \delta) \cdot m_{i}+\operatorname{trans}(\alpha, \theta, \delta)\right)\right\|^{2}
$$

where $\left\{e_{j}\right\}$ are the detected edge pixels of the DLHs; $\left\{m_{i}\right\}$ are the points used to describe the geometrical models the DLHs; $P()$ denotes the projection operator; $N()$ denotes the action of finding the closest edge pixel of the simulated projection point into the image of a model point.

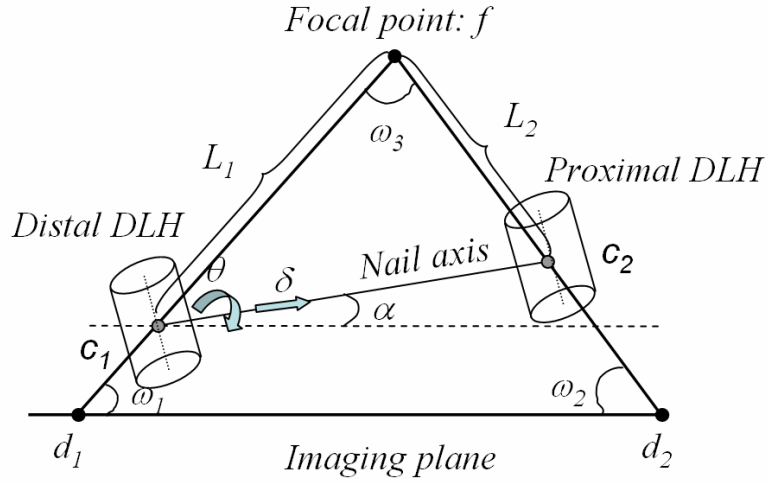

Figure 3. Schematic view of model-based fitting

\subsection{Variable Decomposition Approach}

The control variables in equation (4) can be decomposed into two sets: (a) the angle $\alpha$ between the nail axis and its projection in the imaging plane; and (b) the rotation and translation distance of the geometrical models of the DLHs along the nail axis $(\theta, \delta)$. Now the original optimization problem can be re-formulated as:

$$
\left.\min _{\alpha^{*}}\left[\min _{\left\{\theta^{*}, \delta^{*}\right\}} \sum_{i}\left\|e_{j=N(i)}-P\left(\operatorname{rot}(\alpha, \theta, \delta) \cdot m_{i}+\operatorname{tran}(\alpha, \theta, \delta)\right)\right\|^{2}\right)\right]
$$

Where the term in the square brackets simply means the minimum sum of distance for a fixed $\alpha$ and all possibilities of $(\theta, \delta)$. The advantage of such decomposition lies in the fact that the latter set of variables can be calculated by using an iterative closest projection point algorithm as proposed in [6] and described below for completeness, which then reduces the original multiple-dimensional optimization problem to a one-dimensional search along a finite interval.

\subsection{The Iterative Closest Projection Point Algorithm}

Given a fixed $\alpha$, we can estimate the positions of both centers of DLHs and the orientation of the nail axis. Then, the initial transformation between the local COS of the geometrical model of the DLHs and A-COS can be obtained by taking the estimated center as the origin, the estimated nail axis as the $u$ axis, and the normal of the imaging plane as the $v$ axis. All points defined in the local COS of the geometrical model of the DLH can then be transformed to $A$-COS using this transformation. The optimal values of the rotation $\theta$ and the translation $\delta$ of the models along the nail axis can be optimally estimated by fitting the geometrical models of the DLHs to the image $[5,6]$.

Let's denote $E$ be a set of $N_{E}$ detected 2D edge pixels $\left\{e_{1}, e_{2}, \ldots, e_{N_{E}}\right\}$ of the DLH projection. Further denote $M^{t-1}$ be a set of $N_{M}$ model point $\left\{m_{0}^{t-1}, m_{1}^{t-1}, \ldots, m_{N_{M}}^{t-1}\right\}$ at iteration step $t-1$. Now in the iteration step $t$, we perform following steps:

Simulating $X$-ray projection: In this step, we simulate the X-ray projection of the geometrical model of the DLH to remove invisible points. Let $P^{t-1}$ be a set of $N_{P} 2 \mathrm{D}$ projection points $\left\{p_{1}^{t-1}, p_{2}^{t-1}, \cdots, p_{N_{P}}^{t-1}\right\}$ obtained by simulating $\mathrm{X}$-ray projection of 3D model into the image. Normally $N_{P}<<N_{M}$. Thus, for each 2D projection point $p_{i}^{t-1}$, we know its associated 3D model point $m_{i}^{t-1}$.

Find closest projection point: In this step, we try to find the closest neighbor edge pixel $e_{i}$ of each 2D model projection point $p_{i}^{t-1}$. 
Establishing 3D-2D correspondence: For each 2D matched pairs $\left(e_{i}, p_{i}^{t-1}\right)$, calculate the forward projection ray $B P_{i}$ of the $2 \mathrm{D}$ edge pixel $e_{i}$. Then for the ray $B P_{i}$, calculate a 3D point pair $P P_{i}^{t-1}=\left(b e_{i}^{t-1}, m_{i}^{t-1}\right)$, where $b e_{i}^{t-1}$ is a point on the line $B P_{i}$ that is closest to the $3 \mathrm{D}$ model point $m_{i}^{t-1}$ of the model projection point $p_{i}^{t-1}$.

Estimating pose: For all calculated 3D point pairs $P P S^{(t-I)}=\left\{P P_{i}^{t-I}\right\}$, find an optimal local solution of all pose parameters by minimizing following cost function:

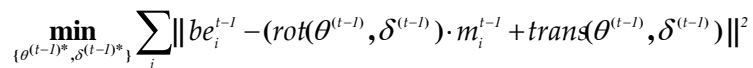

where we drop the symbol $\alpha$ from the expressions of $\operatorname{rot}()$ and $\operatorname{trans}()$, as its value is fixed.

Updating pose: Update the pose of all model points $\left\{m_{1}^{t-1}, m_{2}^{t-1}, \ldots, m_{N_{M}}^{t-1}\right\}$ using the calculated transformation.

These steps are repeated until all pose parameters are converged.

\section{EXPERIMENTAL RESULTS}

We design and conducted in vitro experiments to analyze the accuracy and robustness of the proposed approach. A SYNTHES® (STRATEC Medical, Oberdorf, Switzerland) $9 \mathrm{~mm}$ solid titanium femoral nail was used in our study. It was inserted into a cadaveric human femur and was locked proximally. A Siemens ISO-C C-arm (Siemens AG, Erlangen, Germany) was used to acquire fluoroscopic images for our experiments. The ground truth of the positions of the DLHs was obtained after image acquisition by inserting a custom-made steel rod through the hole and then digitizing both top and bottom centers of the rod using an opto-trackable sharp pointer (OPTOTRAK 3020, Northern Digital Inc, Waterloo, Canada).

Three images acquired from different view directions were used in our experiments, as shown in Fig. 4. For each image, we applied the proposed approach ten times to estimate the poses of the DLHS. The estimated results were compared to the ground truth to compute the error for each DLH, which was defined as the angular difference between the estimated axis of the DLH and the axis obtained through pointer-based digitization.

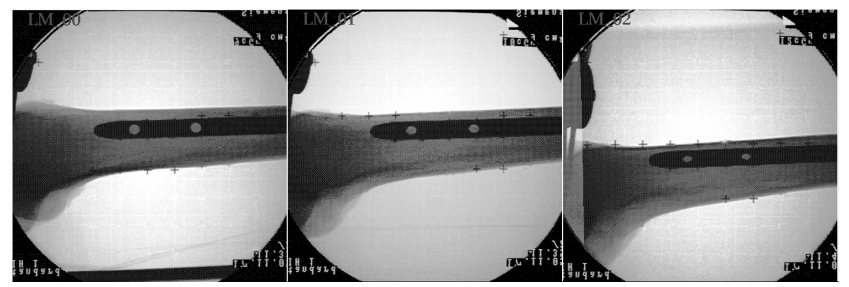

Figure 4. Three images used in our experiments. From left to right: LM_00, LM_01, and LM_02
Table 1. Experimental results

\begin{tabular}{c|c}
\hline Test image & Angular differences $\left(^{\circ}\right)($ mean \pm std $)$ \\
\hline LM_00 & $0.7 \pm 0.3$ \\
\hline LM_01 & $0.9 \pm 0.2$ \\
\hline LM_02 & $1.5 \pm 0.2$ \\
\hline Overall & $1.0 \pm 0.4$ \\
\hline
\end{tabular}

In all studies, the positions and orientations of DLHs could be automatically recovered. The angular errors are shown in Table I. Compared to ground truth, the average angular error was found to be $1.0^{\circ}\left(\operatorname{std}=0.4^{\circ}\right)$.

\section{CONCLUSIONS}

We have presented a novel variable decomposition approach for automatic pose recovery of distal locking holes from single calibrated fluoroscopic image. Unlike previously introduced method [3], our approach does not ask for an image with perfectly circular holes. Our in vitro experimental results demonstrate that the accuracy of our approach is adequate for successful distal locking of intramedullary nails.

\section{REFERENCES}

[1] C. Krettek., J. Mann $\beta$, T. Miclau, et al. Deformation of femoral nails with intramedullary insertion. J Orthop Res, 16(5): $572-575,1998$.

[2] S. Skjeldal and S.Backe. Interlocking medullary nails radiation doses in distal targeting. Arch Orthopaedic Trauma Surg, 106: 179 - 181, 1987.

[3] Y. Zhu, R.Phillips, J.G.Griffiths, et al. Recovery of distal hole axis in intramedullary nail trajectory planning. Proc Inst Mech Eng [H], 216(5): 323 - 332, 2002.

[4] Z. Yaniv and L.Joskowicz. Precise robot-assisted guide positioning for distal locking of intramedullary nails. IEEE T Med Imaging, 24(5): 624 - 635, 2005.

[5] G. Zheng, et al. A robust and accurate two-stage approach for automatic recovery of distal locking holes in computer-assisted intramedullary nailing of femoral shaft fractures. Submitted to IEEE Transactions on Medical Image, 2006.

[6] G. Zheng, X. Zhang, and L.-P. Nolte. Automatic pose recovery of the distal locking holes from single calibrated fluoroscopic image fro computer-assisted intramedullary nailing of femoral shaft fractures. In Proceedings of the third international workshop on medical imaging and augmented reality (MIAR 2006), LNCS Volume 4091, pp. 195-202, 2006.

[7] R. Hofstetter, M. Slomczykowski, M. Sati, and L.-P. Nolte. Fluoroscopy as an imaging means for computer-assisted surgical navigation. Comp Aid Surg, 4: 65 - 76, 1999.

[8] T. Risse, Hough transform for line recognition, Computer Vision and Image Processing. 46: 327-345, 1989. 